\title{
Integridad y desprecio* \\ Motivos básicos de una concepción de la moral desde la teoría del reconocimiento
}

\author{
AXEL HONNETH \\ Universidad de Constanza
}

Si se admite con Enst Bloch que la completa integridad del hombre, su dignidad, sólo se alcanza estableciendo una adecuada protección ante los distintos modos de ofensa y de desprecio personal, entonces eso significa a la inversa que la integridad de la persona humana depende constitutivamente de la experiencia de reconocimiento intersubjetivo. El presente texto quiere aclarar esa conexión, premisa pro- pia de una teoría normativa del reconocimiento recíproco, entre desprecio e integridad humana que Bloch sugirió pero no desarrolló, para así poder introducir una tesis fuerte de sociología moral: la experiencia del desprecio personal representa un impulso moral en el proceso de desarrollo de la sociedad, pues el progreso moral es el resultado de la lucha por el reconocimiento.

Ernst Bloch intentó mostrar en su libro Derecho natural y dignidad humana que en todas las diferentes prolongaciones de la tradición iusnaturalista existía el núcleo de una intuición moral única. Como es sabido, el resultado que obtuvo su todavía hoy fascinante investigación consiste en la tesis de que, a diferencia de la perspectiva eudemonista de las utopías sociales, el Derecho Natural se orienta a la protección de la dignidad humana. Con el lenguaje sobrio de las distinciones analíticas, que carece de aquella normalmente típica expresividad de Bloch, se dice en un lugar destacado del libro: «Las utopías sociales se dirigen en su mayor parte a la felicidad o, por lo menos, a la eliminación de la necesidad y de las circunstancias que la conservan o la producen. Las teorías del Derecho Natural [...] se dirigen predominantemente a la dignidad, a los derechos humanos, a las garantías jurídicas de la seguridad o la libertad humanas, en tanto que categorias del orgullo humano. Conforme a lo cual, la utopía social se dirige ante todo a la eliminación de la miseria humana, mientras el Derecho Natural ante todo a la eliminación de la humillación humanaw.' Las formulaciones negativas que Bloch emplea en esta última frase nos permiten conocer las dos consideraciones principales que introduce en su argumentación como premisas determinantes: en primer lugar, que la sustancia de todo aquello que en teoría moral se llama «dignidad hu-

* Traducción de Juan Carlos Velasco Atroyo. 
mana» sólo se constituye por la vía indirecta de una determinación de los modos de la humillación y el daño personal; $\mathrm{y}$, en segundo lugar, que tales experiencias negativas de desprecio y ofensa, que eran sólo la fijación de un fin normativo de aseguramiento de la dignidad humana en el proceso histórico, también desarrollan un impulso práctico. En la primera premisa está formulada ciertamente una tesis de filosofía moral; en la segunda tesis, si se puede decir así, una de sociología moral. Por cierto que ambas premisas guardan en Bloch el carácter de hipótesis no dilucidadas filosóficamente, porque él las ha usado sólo como expediente teórico para una apropiación marxista de la tradición iusnaturalista. Aunque forman el centro teórico moral de su argumentación, no las ha hecho ya objeto de una reflexión expresa en el marco hermenéutico de la investigación. De esta manera se le ha mantenido oculto aquello que representaba filosóficamente el punto propio de su libro: si un concepto de la dignidad del hombre, de su completa integridad, se logra sólo aproximativamente en el camino de una determinación de los modos de la ofensa personal y el desprecio, entonces eso significa a la inversa que la integridad de la persona humana depende constitutivamente de la experiencia de reconocimiento intersubjetivo. Bloch se sirve, sin saberlo, de una teoría normativa del reconocimiento recíproco. De ahí que la integridad del sujeto humano, porque puede llegar a ser herido por la ofensa y el desprecio, se verá en dependencia del beneplácito y aprecio de otras personas. En lo siguiente quiero aclarar un poco más esa conexión determinada negativamente, que ha sido perfilada así, entre desprecio e integridad humana, que Bloch ha sugerido pero no ha desarrollado. Así, en conexión con la primera premisa de su investigación, pretendo en primer lugar distinguir por de pronto unas maneras de desprecio personal de otras (I). De la distinción entre las tres formas elementales de desprecio resultará después indirectamente, en el siguiente paso, un examen de la totalidad de experiencias de reconocimiento que el hombre necesita si ha de ser protegido en su integridad (II). Sólo después de esta pieza intermedia de teoría del reconocimiento puedo abordar a continuación la segunda premisa: la premisa de sociología moral introducida por Bloch, en la que se afirma que la experiencia del desprecio personal representa un impulso moral en el proceso de desarrollo de la sociedad (III).

\section{I}

En nuestro uso cotidiano del lenguaje está todavía asumido como un saber evidente que la integridad del hombre se debe de modo subconsciente a la aprobación o al reconocimiento de los otros sujetos. En la autodescripción de los que se ven tratados moralmente de un modo falso juegan hasta hoy un papel dominante categorías tales que, como las de "ofensa" o "humilla- 
ción", se refieren a formas de desprecio, de reconocimiento negado. Mediante conceptos negativos de esta índole se designa una conducta que no representa ya tanto una injusticia, dado que menoscaba a los sujetos en su libertad de acción o les ocasiona daños. Más bien se refiere a aquel aspecto de una conducta dañina por el que las personas son heridas en la comprensión positiva de sí mismas que han adquirido por vías intersubjetivas. Aquellos conceptos de «desprecio» $\mathbf{u}$ "ofensa» en modo alguno se han de emplear, con sentido, sin la referencia implícita a las exigencias que un sujeto plantea a la reacción reconocedora de su congénere. De ahí que nuestro uso cotidiano del lenguajc, que Bloch también comparte como evidente, contenga la referencia a un concepto de la teoría de la intersubjetividad, en el que la invulnerabilidad y la integridad del ser humano han de ser consideradas en dependencia con la aprobación de los otros. En la doctrina hegeliana del reconocimiento, pero ante todo en la psicología social de G.H. Mead, este saber intuitivo ha sido transformado dentro del contexto de una teoría sistemática. De ahí se considerará la individualización del hombre como un proceso en el que el individuo puede conseguir una identidad práctica conforme es capaz de cerciorarse del reconocimiento de sí mismo a través de un círculo creciente de interlocutores. ${ }^{2}$ Sujetos capaces de habla y acción sólo se constituirán como individuos aprendiendo a referirse a sí mismos como un ser bajo la perspectiva de otros que les aprueban, como un ser a quien le corresponden ciertas cualidades y capacidades. De ahí forman una conciencia creciente de su individualidad al mismo tiempo que una dependencia progresiva de sus relaciones de reconocimiento que les ofrece el mundo de la vida de su contexto social. De esta delimitación interna de individualización y reconocimiento, de la que tanto Hegel como Mead han partido, resulta aquella especial vulnerabilidad del ser humano, a la que nos referimos mediante el concepto de «desprecios: dado que la autoimagen normativa de cada hombre, su «Me», como diría Mead, depende de la posibilidad del continuo reaseguro en el otro que acompaña a la experiencia de desprecio, existe el peligro de una herida que puede llevar al desmoronamiento de la identidad de la persona completa.

Ahora es ostensible que todo lo que coloquialmente se designa como «desprecio» u "ofensa» parece abarcar grados diferentes de profundidad de la herida psíquica de un sujeto: entre la manifiesta humillación, que se asocia con la privación de los derechos fundamentales más elementales, y el sublime abatimiento que va acompañado de la alusión pública al fracaso de una persona, subsiste una diferencia categorial que amenaza con perderse con el empleo de una única expresión. Ya la circunstancia de que tendamos también a proponer intuitivamente gradaciones en el contraconcepto positivo de "respeto" indica diferencias internas, que existen entre las formas de desprecio: a la introducción de este concepto en la teoría 
moral kantiana se retrotrae una discusión persistente hasta hoy en la que con diferentes medios, sean fenomenológicos o analítico-lingüísticos, se ha emprendido el intento de diferenciar grados de desprecio de otra persona según qué características de personalidad llegan a ser reconocidas en ella y de qué modo. ${ }^{3}$ Me remito implícitamente al curso del debate ahí aludido, cuando emprendo el intento de destacar sistemáticamente cada una de las tres maneras de "desprecio" frente a las demás; sus diferencias deben medirse por el grado en que pueden perturbar la relación práctica de una persona consigo misma privándola del reconocimiento de unas determinadas pretensiones de identidad.

Si se adoptara tal punto de vista como medida de comparación, entonces cobraría sentido partir de un tipo de desprecio que roza el estrato de la integridad física de la persona: aquellas formas de maltrato practicado, en las que a una persona le son retiradas por la fuerza todas las posibilidades de libre disposición sobre su cuerpo, representan la manera más primitiva de humillación personal. El motivo es el siguiente: que cada tentativa, contra la voluntad de una persona e independientemente de la intención, de apoderarse de su cuerpo produce un grado de humillación tal, que afecta de manera destructiva más profundamente que cualquier otra forma de maltrato en la relación práctica consigo mismo. Lo peculiar de estas maneras de vulneración física, como ocurre con la tortura o con la violación, no es que impliquen dolor corporal propiamente dicho, sino su unión con el sentimiento de estar expuesto indefenso a la voluntad de otro sujeto hasta la privación consciente de la realidad. ${ }^{4} \mathrm{El}$ maltrato físico de un sujeto representa un tipo de desprecio que hiere persistentemente la confianza antes aprendida en la capacidad de coordinación autónoma del propio cuerpo. De aquí la consecuencia lógica, junto a una especie de vergüenza social, de pérdida de confianza en sí mismo y en el mundo, pérdida que alcanza niveles físicos del trato con otros sujetos. Por tanto, el reconocimiento de que aquí se priva a la persona por el desprecio es el evidente respeto de aquella disposición autónoma sobre el propio cuerpo, respeto que por su parte sólo ha sido adquirido en la socialización por medio de experiencias de inclinación emocional. La lograda integración entre cualidades corporales y espirituales de comportaniento se romperá ulteriormentc en cierto modo desde fuera, y por ello se destruye persistentemente la forma más elemental de relación con uno mismo: la confianza en sí mismo.

De este tipo extremo de desprecio, que ya interrumpe la continuidad de la imagen positiva de sí mismo en el plano corporal, cabe deslindar aquellas formas de humillación que afectan a la autocomprensión normativa de una persona. Con ello se alude a tipos de desprecio personal que se causan a un sujeto, de manera que quede estructuralmente excluido de la posesión de determinados derechos dentro de una sociedad. Por «de- 
rechos» se entienden aquellas pretensiones individuales con las que puede contar legítimamente una persona para su realización social, dado que participa en su organización institucional con los mismos derechos como miembro pleno de una comunidad. Si se le retienen sistemáticamente ciertos derechos de este tipo, también implica esto la afirmación de que no se le concede en igual medida que a los otros miembros de la sociedad la capacidad de imputación moral. Lo especial en estas formas de desprecio, como acontece en la privación de derechos o en la marginación social, representa no sólo la limitación comparativa de la autonomía personal sino también su conexión con el sentimiento de no poseer el status de sujetos con igualdad moral de derechos y con pleno valor. El hecho de que se preserven las pretensiones jurídicas, socialmente válidas, de no ser dañado en las expectativas intersubjetivas significa, para el individuo, ser reconocido como sujeto capaz de formular un juicio moral; en esa medida, con la experiencia de la privación de derecho se da típicamente también una pérdida de autoestima, de la capacidad de referirse a uno mismo como interlocutor en igualdad de derechos con sus congéneres. ${ }^{5}$ Con lo cual, el reconocimiento de que se priva a una persona por medio del desprecio es el respeto cognitivo de una capacidad de imputación moral, que por su parte sólo ha sido adquirida con gran esfuerzo en procesos de interacción socializadora. Frente a ese segundo tipo de desprecio, que arrastra la autocomprensión normativa de un sujeto a la compasión, se puede establecer aún un último tipo de humillación que se refiere negativamente al valor social de individuos o grupos; sólo con estas formas en cierto modo valorativas del desprecio, de la profanación de la dignidad de los modos de vida individuales o colectivos, se consigue realmente la forma de comportamiento a la cual hoy en día se alude de manera coloquial ante todo con conceptos tales como «ofensa" o «deshonra». Por «honor», "dignidad» o, dicho con un término moderno, por «status» de una persona se entiende la medida de aprecio social que corresponde a su modo de autorrealizarse en el horizonte de la tradición cultural de una sociedad; 6 si esta jerarquía social de valores estuviera constituida de manera que desprestigiara formas de vida o convicciones individuales considerándolas de menos valor o defectuosas, entonces se quitaría al sujeto afectado toda posibilidad de atribuir un valor social a sus propias capacidades. La degradación valorativa de determinados patrones de autorrealización tiene como consecuencia, para su portador, que tales patrones no puedan referirse a la realización de su vida como a algo que dentro de su comunidad tuviera una significación positiva; para el individuo se da también típicamente, junto a la experiencia de una devaluación social semejante, una pérdida de autoestima personal y de la oportunidad, por tanto, de poder comprenderse a sí mismo como un ser apreciado por sus cualidades y capacidades características. El reconocimiento del que la persona se ve aquí privada por el despre- 
cio es la aprobación social a una forma de autorrealización que ella misma tuvo que encontrar fatigosamente con ayuda tan sólo del estímulo creado por la solidaridad del grupo.

Es ahora típico, para los tres grupos de experiencias de desprecio que de esta manera se pueden diferenciar analíticamente uno de otro, que sus consecuencias individuales sean siempre descritas con metáforas que aluden a desgracias para el cuerpo humano: en las investigaciones psicológicas que rastrean las secuelas personales de las experiencias de tortura o de violación, se habla a menudo de "muerte psíquica»; dentro del ámbito de investigación que, tomando como ejemplo la esclavitud, se ocupa del proceso colectivo con que se engendra la privación de derechos y la marginación social, se ha introducido el concepto de «muerte social»; y en referencia al tipo de desprecio que se encuentra en la denigración de la dignidad cultural de una forma de vida, es la categoría de «ultraje» (Kränkung) la que se aplica preferentemente. ${ }^{7}$ Con este tipo de alusiones metafóricas al padecimiento físico y a la muerte se expresa lingüísticamente que a las distintas formas de desprecio por la integridad psíquica del ser humano les corresponde el mismo papel negativo que adoptan las enfermedades orgánicas en relación con la reproducción de su cuerpo: por la vivencia de la humillación y el rebajamiento social, peligran los seres humanos en su identidad tanto como peligra su vida física ante el padecimiento de enfermedades. Si esta relación, indicada por nuestra práctica lingüística, no es totalmente inaceptable, entonces como consecuencia de nuestra sinopsis sobre las diferentes formas de desprecio tendrían que poderse extraer también conclusiones en orden a lo que, por así decir, contribuye a la «salud" psíquica, a la integridad del ser humano; a la prevención de enfermedades correspondería, visto así, la garantía social de relaciones de reconocimiento que sean capaces de proteger lo más ampliamente posible a los sujetos contra la experiencia del desprecio. La tesis ahí indicada quisiera intentar explicarla brevemente en la segunda parte.

Si a la defensa de la integridad humana por medio de la protección frente al menoscabo de la dignidad y a la ofensa la consideramos, al igual que Ernst Bloch, como el motivo moral central que se encuentra detrás de todas las versiones de la tradición iusnaturalista, entonces puede dar un giro positivo el problema que aquí se plantea, pues la diferenciación de tres formas de desprecio, objeto de las anteriores reflexiones, contiene ya la referencia indirecta a aquellas relaciones de reconocimiento intersubjetivo, cuya existencia, en conjunto, constituye el presupuesto para la integridad del ser humano. Los sujetos con capacidad de acción, como Hegel y Mead demostraron convincentemente, deben a la experiencia del reconoci- 
miento recíproco la posibilidad de formación de una relación positiva consigo mismos: su Yo práctico, dado que aprende a tener confianza y respeto en sí mismo desde la perspectiva de la reacción aprobatoria de los interlocutores, está orientado hacia relaciones intersubjetivas en las que puede experimentar el reconocimiento. Pero en estas relaciones de reconocimiento recíproco también hay que efectuar las mismas diferenciaciones que se hallaron en las distintas formas de desprecio social, pues cada una de las diferentes formas de ultraje y menoscabo de la dignidad habia dañado un particular trato de relación positiva con uno mismo cuyo desarrollo formacional manifiestamente sólo puede deberse de nuevo, por su parte, a la existencia de un particular trato de reconocimiento en cada caso. En esa medida, la diferenciación de las tres formas de desprecio nos da una clave para distinguir entre el mismo número de relaciones de reconocimiento mutuo; éstas, si nuestras reflexiones son correctas, establecen la infraestructura moral de un mundo de vida social en el que los individuos pueden no sólo adquirir sino también mantener su integridad como personas humanas.

El punto de partida de mis diferenciaciones lo representaban aquellos tipos de desprecio que se encuentran en las humillaciones físicas tales como la tortura y la violación. Por eso podían servir como forma fundamental de degradación del hombre ya que le privan de la autonomía incorporada en el trato consigo mismo y destruyen con ello una parte de la elemental confianza en el mundo. La relación de reconocimiento que corresponde a esta forma de desprecio, dado que ayuda al individuo en la formación de tal confianza en sí mismo vinculada al cuerpo, es la de una inclinación emocional que el romántico, como Hegel, ha intentado captar con el concepto de "amor". Como las necesidades y los afectos en cierto modo sólo pueden obtener "confirmación", en definitiva, siendo directamente satisfechos o correspondidos, el reconocimiento mismo tiene que poseer aquí el carácter de aprobación afectiva y de estímulo; en esa medida esta relación de reconocimiento está también vinculada a la existencia corpórea de otros concretos que se dispensan mutuamente sentimientos de estima. La actitud positiva que cabe al individuo adoptar para consigo mismo cuando experimenta un reconocimiento afectivo de este tipo es la de la confianza cn sí mismo; con esto se alude al estrato fundamental de una seguridad emocional y corpórea en la expresión de las propias necesidades y sensaciones, que consituye el presupuesto psíquico para el desarrollo de todas las demás actitudes de autoestima. ${ }^{8}$ No hay que generalizar este modo de reconocimiento recíproco más allá del círculo de las relaciones sociales primarias como es el caso de las vinculaciones afectivas que siguen el patrón de las familias, amistades o relaciones amorosas. Dado que las actitudes de afirmación emócional están vinculadas a los requisitos individualmente no disponibles de simpatía y atracción, no se pueden 
transferir a voluntad a un número mayor de interlocutores. De aquí se desprende que a esta relación de reconocimiento es inherente un particularismo moral que no se puede deshacer por medio de ningún intento de generalización.

Del maltrato físico, que corresponde positivamente a la favorable inclinación emocional en relaciones primarias de este tipo, se pudo diferenciar como una segunda forma de desprecio la privación de derechos y la marginación social; con este desprecio se degrada a un ser humano de manera que no se le concede en el interior de su comunidad social la capacidad de responsabilidad moral propia de una persona con plenitud de derechos. De acuerdo con ello, este tipo de desprecio debe corresponderse con una relación de reconocimiento recíproco en la que el individuo aprende a comprenderse desde el punto de vista de las personas con las que se interrelaciona como un portador igual de derechos; como mecanismo por el que esto acontece identificó G.H. Mead el proceso de adopción de la perspectiva de un "otro generalizado", proceso que garantiza al sujeto (Selbst) de la relación práctica consigo mismo (Selbstbeziehung), junto con la imposición de ciertas obligaciones, a la vez la satisfacción de determinadas exigencias. A esta relación de reconocimiento corresponde, como se deduce de ello, un carácter primariamente cognitivo al contrario de lo que acontece en las relaciones íntimas: ego y alter se reconocen mutuamente como personas sujetos de derechos porque poseen un saber común de aquellas normas que regulan los derechos y deberes que, por así decir, les competen en el marco de la comunidad en cada caso. La actitud positiva que puede adoptar un sujeto para consigo mismo cuando experimenta este reconocimiento jurídico es la de una elemental autoestima; le cabe considerarse como una persona que comparte con todos los demás niembros de su comunidad las características de un actor moralmente responsable. ${ }^{9}$ La relación jurídica permite, al contrario que la relación de reconocimiento que se da en la relación primaria, una generalización del medio de reconocimiento que le es propio, en dos direcciones: una expansión de contenido y otra social de los derechos. En el primer caso, el derecho gana en contenidos materiales, con los que de manera progresiva consiguen consideración jurídica las diferencias de oportunidades individuales para la realización de las libertades garantizadas intersubjetivamente. En el segundo caso, al contrario, se universaliza la relación jurídica, en el sentido de que a un círculo creciente de grupos hasta ahora marginados o perjudicados le son reconocidos los mismos derechos que al resto de los miembros de la comunidad. De ahí que a la relación de reconocimiento por medio del derecho le sea inherente un universalismo de principios que alcanza su desenvolvimiento a través de luchas históricas.

Finalmente, el tercer tipo de desprecio - tras el que iba en mi propuesta de diferenciación - consistía en la degradación del valor social de 
las formas de autorrealización; estas muestras de degradación valorativa de determinadas formas de vida tienen como consecuencia para los sujetos afectados el no poderse referir positivamente, en el sentido del aprecio social, a sus capacidades adquiridas a lo largo de la propia historia vital. De ahí que la relación de reconocimiento que corresponde a esta forma de desprecio sea la que pueda ayudar al individuo a una autoestima de este tipo, una relación de aprobación solidaria para formas de vida alternativas; en esta relación encontrarían los sujetos, con sus características individuales, como personas individualizadas a lo largo de su biografía personal, el reconocimicnto recíprocamente alentador. Mead argumentó, con la mirada puesta en tal relación de reconocimiento: dado que el sujeto (Selbst) de la autorrelación práctica (Selbstbeziehung) tiene que cerciorarse de sí no sólo en tanto que ser autónomo sino también como ser individual, tiene que poder situarse además en la perspectiva de un «otro generalizado» que le proporcione aprobación intersubjetiva en su pretensión de unicidad e insustituibilidad; $;^{10}$ de la posibilidad de tal autoconfirmación ética se cuida una relación de reconocimiento mutuo, en la que ego y alter se encuentran dentro del horizonte de unos valores y metas que les señalan recíprocamente el irrenunciable significado de la propia vida de cada uno para el correspondiente otro. Mientras esta forma de reconocimiento tenga que presuponer la experiencia vital de cargas y responsabilidades compartidas, también estará siempre involucrado en ella, más allá del momento cognitivo de un saber ético, un elemento afectivo de participación solidaria. La actitud positiva que puede adoptar un sujeto consigo mismo cuando se le reconoce de esta manera es la de una valoración de la propia persona: dado que es respetado por sus interlocutores como una persona biográficamente individualizada, puede identificarse el sujeto sin restricciones con sus cualidades especiales y sus capacidades. Lo especial de esa relación ética de reconocimiento es también para Mead la circunstancia de que esto apunta íntimamente a la posibilidad de una sucesiva apertura a las tendencias realizadoras del sujeto (Selbst): las normas éticas, a cuya luz se pueden reconocer mutuamente los individuos en su particularidad individual, están abiertamente a favor de un proceso de ruptura con la tradición, en el curso de la cual pierden su carácter jerarquizador y prescriptivo por su generalización creciente. De aquí que esta relación de reconocimiento de la solidaridad o de la eticidad (Sittlichkeit) conlleve un principio de diferencia igualitaria, que puede lograr su desenvolvimiento bajo la presión de los sujetos individualizados.

Con estos tres patrones de reconocimiento (el amor, el derecho y la solidaridad) quedan establecidos aquellos requisitos formales de las relaciones de interacción en cuyo contexto pueden los seres humanos sentirse seguros de su «dignidad» o integridad. Las mencionadas determinaciones son formales, porque con ayuda de los tipos de reconocimiento sólo pue- 
den perfilarse características estructurales de los modos de comunicación, pero no intentan establecer además sus formas de realización institucional; asimismo, con la enumeración de los tres patrones de reconocimiento quedan también mencionadas las infraestructuras morales que tienen que pertenecer a un mundo de vida social si ha de proteger a sus miembros. De acuerdo con esto, aquí «integridad" también es capaz de significar que un sujeto puede saberse apoyado por la sociedad en todo el espectro de sus relaciones prácticas consigo mismo; si participa en un mundo de vida social en el que quepa encontrar escalonados esos tres patrones de reconocimiento, sea cual sea su forma de concreción, puede referirse a sí mismo mediante los modi positivos de la autoconfianza, la autoestima y el autoaprecio. Para la tesis de filosofía moral que ha desarrollado Bloch en su gran investigación, surge de estos razonamientos, con todo, la posibilidad de una reformulación positiva: la moral defiende - si se la toma como institución para proteger la dignidad humana - la reciprocidad del amor, la universalidad de los derechos y el igualitarismo de la solidaridad contra la entrega a la violencia y a la opresión; con otras palabras, le es propio un interés por el despliegue de aquellos principios que subyacen estructuralmente a las diversas formas de reconocimiento. Ahora bien: que un elemento de la realidad social deba favorecer, en el proceso histórico, a una moralidad así entendida es la segunda premisa de sociología moral en la investigación de Bloch; en ella quisiera entrar todavía, en la última parte de mis reflexiones, con unas cuantas citas paradigmáticas.

\section{III}

Bloch ha llamado la atención en diferentes lugares de su libro - nunca sin embargo sin el pathos que le es propio- sobre las fuentes morales de motivación de los cambios sociales: sin la sensación añadida de la dignidad herida -así consta allí - la mera experiencia de necesidad económica y dependencia política nunca se hubiera convertido históricamente en una fuerza impulsora de los movimientos subversivos prácticos; a la escasez económica o a la opresión social siempre hubo que añadir el sentimiento de ser despreciados en la exigencia de integridad de la propia persona, antes de que pudieran convertirse en motivo inductor de los levantamientos revolucionarios. ${ }^{11}$ Con reflexiones de esta índole, para las que en su investigación apenas se pueden localizar justificantes históricos, Bloch ha pretendido resaltar una disposición moral en el hombre, disposición que favorece la moral del Derecho Natural definida por él negativamente: no es en fuentes positivas de motivación, como el altruismo o el respeto, donde la moral puede encontrar un apoyo práctico dentro de la realidad social, sino en la experiencia del desprecio social, experiencia que se presenta espontánea y reiteradamente. En estas reflexiones me parece a mí apunta- 
da la referencia a una construcción con la cual se puede allanar una pizca más el déficit en la tcorización de las motivaciones que hay en la teoria moral contemporánea; ${ }^{12}$ en todo caso, esa construcción presupone que las determinaciones de los cometidos de la moral están más directamente arraigadas en las pretensiones intersubjetivas de los sujetos corpóreos de lo que acontece tal vez hoy en una fundamentación de la moral desde tcorias lingüísticas.

Los seres humanos, remitiéndonos por última vez a la visión de Hegel y Mead, están necesitados del reconocimiento intersubjetivo de sus capacidades y obras para poder lograr así una relación satisfactoria consigo mismos. Si faltara esa forma de aprobación social, en cualquier nivel de su desarrollo, se abriría, por así decir, un vacío psíquico en su personalidad que buscaría expresarse mediante reacciones afectivas de índole negativa como la vergüenza o la ira, el ultraje o el menosprecio. De ahí que la experiencia del desprecio vaya acompañada siempre de sensaciones afectivas que pueden revelar al individuo, por principio, que le son negadas determinadas formas de reconocimiento social. Para hacer plausible esta compleja tesis, al menos a grandes rasgos, se recomienda conectarla con un concepto de los sentimientos humanos que se remite a la psicología pragmatista de John Dewey.

En algunos escritos tempranos, Dewey se había opuesto a la interpretación, ampliamente extendida, según la cual la suscitación de sentimientos en los seres humanos se debía comprender como forma expresiva de estados de ánimo internos. Quería mostrar que, partiendo de una concepción de este tipo -también cabe encontrarla en William James-, se desconoce forzosamente la función de los sentimientos referida al comportamiento, pues siempre se presupone el acontecer psíquico como un "interior" para las acciones dirigidas hacia «fuera».13 El punto de partida de la argumentación de Dewey representa la observación de que los sentimientos sólo aparecen, al fin y a la postre, en el horizonte de vivencias del hombre dependiendo positiva o negativamente de la ejecución de los actos, a saber: o bien acompañan, en tanto que estados de excitación vinculados con el cuerpo, a la experiencia de "comunicaciones" especialmente satisfactorias (con cosas o personas), o bien surgen como vivencias de rechazo por la ejecución de actos fallidos o impedidos. El análisis de tales vivencias de rechazo le pone a Dewey en la mano la llave que le permite alcanzar una concepción de los sentimientos humanos desde la teoría de la acción. Como consecuencia de esta concepción, los sentimientos negativos tales como ira, indignación y pena constituyen el lado afectivo de aquella desviación atencional hacia las expectativas propias, desviación que ha de presentarse en el momento en que tras haber realizado una acción no puede encontrarse la correspondencia planeada; con sentimientos positivos como alegría u orgullo reacciona sin embargo el sujeto cuando se le libera de 
golpe de un estado de excitación gravoso, porque ha podido encontrar para un problema apremiante de comportamiento una solución adecuada y afortunada. En resumen, los sentimientos representan, según Dewey, las reacciones afectivas en el rechazo del éxito o del fracaso de nuestras intenciones de comportamiento.

De este punto de partida general se abre un camino para nuevas diferenciaciones si se distinguen con más exactitud los tipos de "perturbaciones» que pueden, por principio, hacer fracasar el comportamiento habitual del hombre. Dado que perturbaciones o fracasos de este tipo se miden siempre por las expectativas que preceden orientativamente a la ejecución de un acto, se ofrece una primera subdivisión grosso modo según dos tipos diferenciables de expectativas: el comportamiento rutinario de los seres humanos puede chocar con impedimentos o bien en el marco de las expectativas instrumentales de éxito o en el marco de las expectativas normativas de comportamiento. Si fracasan acciones orientadas al éxito, a causa de resistencias que les salen al paso imprevisiblemente en el campo de cometidos a dominar, conduce esto a perturbaciones «técnicas" en el más amplio sentido; por el contrario, si hay situaciones en que fallan acciones reguladas por normas porque resultan éstas dañadas en tanto que normas válidamente supuestas, entonces esto conduce a conflictos "morales» en el mundo de vida social. Este segundo grupo de ejecución frustrada de actos constituye el horizonte empírico en que las reacciones afectivas morales del hombre tienen su sede práctica; cabe entender, en el sentido de Dewey, a los sentimientos morales como excitaciones emocionales con las que reaccionan los seres humanos cuando experimentan un rechazo imprevisto de su comportamiento debido a una lesión en las expectativas normativas de conducta. Las diferencias entre las reacciones afectivas particulares se miden, de modo enteramente elemental, tomando en cuenta si la lesión de la norma - lesión que es lo que frena al acto - viene causada por el propio sujeto agente o por sus interlocutores: en el primer caso se trata de sentimientos de culpa, en el segundo de afectos de indignación moral con los cuales la persona experimenta el rechazo de sus actos. Pero en ambos casos es válido lo que Dewey consideró como típico de estas situaciones de vivencia afectiva de un comportamiento que se rechaza, a saber: que, junto con la desviación de la atención hacia las expectativas propias, se hacen a la vez conscientes sus elementos cognitivos (aquí, por tanto, el saber moral), por los cuales se había dejado guiar el comportamiento planeado y ahora frenado.

De los sentimientos morales es la vergüenza el que posee el carácter más abierto, siempre y cuando no se entienda por ella sólo el miedo -claramente de un arraigo antropológico profundo- a la mera exposición del propio cuerpo; en el caso de la vergüienza no queda establecido de antemano desde cuál de los lados de la interacción se infringe la norma moral que 
falta, por así decir, al sujeto para la rutinaria continuación de su comportamiento. El contenido afectivo de la vergüenza consiste ante todo -tal como las posturas psicoanalíticas y fenomenológicas concuerdan en mantener - en una especie de hundimiento de la propia sensación de autoestima; el sujeto que, con la vivencia del rechazo de su comportamiento, se avergüenza de sí mismo, se siente de un valor social menor del que había supuesto anteriormente; esto, considerado desde el punto de vista psicoanalítico, significa que por la paralizadora lesión de una norma moral no se ve afectado aquí negativamente el super-ego, sino el ideal del ego de un sujeto. ${ }^{14}$ Este tipo de vergüenza que sólo se vive en presencia de los otros sujetos de interacción reales o imaginarios, sobre los que en cierto modo recae el papel de testigos del lesionado ideal del ego, puede de nuevo deberse al propio sujeto como causa de ella o puede achacarse a la culpa ajena: en el primer caso el sujeto se experimenta a sí mismo, por ello, como de menos valor, porque ha infringido una norma moral cuyo cumplimiento constituía un fundamento de su propio ideal del Yo; en el segundo caso, sin embargo, el sujeto resulta humillado por una sensación de falta del propio valor debido a que los sujetos con los que se interrelaciona violan nomas morales cuyo cumplimiento ha permitido considerarle como la persona que él, de acuerdo con sus ideales del Yo, desea ser. Y aquí se desata la crisis moral en la comunicación, al resultar defraudadas expectativas que el sujeto agente creyó poder transferir a la disposición respetuosa de su prójimo. En tal medida, este segundo tipo de vergüenza moral representa la suscitación de un sentimiento que domina al sujeto cuando, a causa de la experiencia de un desprecio de sus pretensiones como un Yo, sencillamente ya no es capaz de seguir actuando. Lo que descubre de sí mismo a través de esta sensación es la constitutiva dependencia de su propia persona del reconocimiento de los otros.

En esta reacción afectiva de vergüenza tiene, por ello, un débil asidero empírico una moral que intenta hacer valer los principios del reconocimiento recíproco. Dentro de tales principios, se ve facultada para pedir la palabra una vez y otra, renovadamente, la convicción cognitiva de que lo que sucede en la praxis del desprecio causa daños a las condiciones intersubjetivas de la socialización humana. Sólo porque los sujetos humanos no pueden reaccionar de manera afectivamente neutra ante ultrajes sociales como son el maltrato físico, la privación de derechos y la degradación, tienen también una cierta oportunidad de realizarse dentro del mundo de vida social los principios de una moral concebida como teoría del reconocimiento, pues toda reacción afectiva de carácter negativo que se sigue de la experiencia de un desprecio a las pretensiones de reconocimiento, contiene en sí de nuevo la posibilidad de que se patentice también cognitivamente al sujeto afectado la injusticia que se le infligió.

Las diferentes conmociones morales de carácter afectivo con que los 
seres humanos reaccionan frente a la ofensa y al desprecio contienen dentro de sí, de este modo, la oportunidad para una idealizadora anticipación de relaciones de reconocimiento logrado y no distorsionado. Cuán débil es, en verdad, este asidero práctico de la moral dentro de la realidad social, lo demuestra el hecho de que en tales reacciones afectivas la injusticia del desprecio no "tiene que" manifestarse, sino que precisamente sólo "puede» hacerlo: que el potencial cognitivo inherente a los sentimientos de vergüenza social y humillación se convierta en una convicción moral depende en gran parte de en qué condiciones se encuentre el entorno político-cultural de los sujetos afectados. Para elegir una respuesta afirmativa tiene que existir el medio de articular un movimiento social, si es que la experiencia del desprecio debe llegar a ser una fuente motivadora de acciones de resistencia política. Pcro una vez que la condena - fundada en nuestros afectos-- del desprecio y la ofensa ha adoptado la forma de una hucha social, entonces esa condena representa un interés empírico que se corresponde con el objetivo teórico de la moral. De ahí que una concepción de la moral desde la teoría del reconocimiento no puede prescindir de la asistencia de investigaciones históricas y sociológicas capaces de mostrar que el progreso moral es el resultado de una lucha por el reconocimiento.

\section{NOTAS}

1. Bloch, Enst: Naturrecht und merzschliche Wirde, Francfort, 1961, p. 234. [Hay traducción castellana de Felipe González Vincén, Madrid, Aguilar, 1980, p. 209, N. del T.]

2. He intentado reconstruir este concepto de un modo más prolijo en mi escrito de habilitacion (Honneth, Axel: Kampf tum Anerkennung. Ein Theorieprogranm in Anschluss an Hegel zmad Mead, Habilitationsschrift, Francfort. [E] término Kommunikationsparther se ha traducido a lo largo de este artículo como einterlocutor"; de cualquier modo el vocablo alude al socio o compañero con el que se establece una comunicación. $N$. del T.]

3. Cfr. Gurwitsch, Aron: Zur Geschichte des Achtungsbegriffs und zur Theorie der sittichen Gefithle, Inaugural-Dissertation. Wurzburgo, 1987; von lhering, Rudolph: Der Zweck im Recht, Vol. II, Leipzig, 1905, pp. 388 y ss.; hoy con medios de análisis lingüistico: Darwall, Stephen L.: "Two Kinds of Respect», en Ethics, vol. 88, ${ }^{\circ}{ }^{\circ} 1$, pp. 36 y ss.

4. Sobre la privacion del sentido de la realidad mediante la tortura, puede consultarse un excelente trabajo: Scarry, Elaine: The Body in Pain. The Making and Unmaking of the World, Nueva York/Oxford, 1985, cap. 1; una sipnosis de la investigación se puede encontrar en Fran. kenberg, Günther: "Politisches Asyl —in Menschenrecht?", en Kritische Justiz.

5. Sobre la relación entre derechos y autorrespeto cfr. Feinberg, Joel: aThe Nature and Value of Rights», en Rights, Justice and the Bounds of Liberty. Essays in Social Philosophy, Princeton, 1980, pp. 143 y ss.; ahora de un modo más analítico: Wildt, Andreas: Recht und Selbstachtung, Ms. 1990.

6. Entre una inabarcable literatura, para mi la aportación más clarificadora: Korff, Wilhelm: Ehtre, Prestige, Gewissen, Colonia, 1966. Bajo el punto de vista sociológico resulta interesante: Berger, Peter: "On the Obsolescence of the Concept of Honor", European Joumal of Sociology, 11 (1970), p. 339; Speier, Hans: "Honor and Social Structure", en Id.: Social Order and the Risks of War. Papers in Political Sociology, Nueva York, 1952, pp. 36 y ss.

7. En la dirección de una categoría de «muerte psiquica" son acertadas, v.gr, las investi- 
gaciones de Bruno Bettelheim. ofr. Erziehung zum Uberleben. Zur Psychologie der Extremsituation, Munich, 1982, principalmente Parte I; sobre la categoria de emuerte socialn cfr. entre otros: Patterson, Orlando: Slavery and Social Death. A Comparative Study, Cambridge/Mass., 1982; Meillassoux, Claude: Anthropologie der Sklaverei, Francfort, 1989, Parte I, cap. V.

8. Sistemáticamente sobre kautoconfianza" como resultado de tempranas experiencias infantiles de caniño cfr. Erikson, Erika H.: Identität und Lebenszykius, Francfort, 1974, especialmente pp. 62 y ss.; y ya en el contexto de una teoría del reconocimiento orientada conceptualmente a Hegel, pero desarrollada psicoanalíticamente, ofr. Benjanin, Jessica: The Bonds of Love. Psychoanalysis, Feminism, and the Probtem of Domination, Nueva York, 1988, especialmente cap. 1.

9. Sobre este tema cfr. Mead, G.H.: Geist, Identitat und Gesellschafi, Francfort, 1973, p. 236 y ss. [Hay traducción castellana en Buenos Aires, Paidós, 1972, N. del $T$.] Para la reconstrucción bajo el punto de vista del wautorrespeto ofr. Tugendhat, Ernst: Selbstbeurusstsein und Selbstbestimmung, Francfort, 1979, Lección 12, pp. 282 y \$s.

10. Sobre este tema ffr. Mead, G.H.: Geist, Identität und Gesellschaft, o. cit., pp. 244 y ss. Mi propia reconstrucción apunta en esa misma dirección (Honneth, Axel: Kampf um Anmerkennung, o. cit., especialmente pp. 183 y ss.).

11. Cfr. Bloch. Emst: Naturrecht und menschaliche Würde, o. cit., p. 309.

12. Además, y con la vista puesta en Max Horkheimer: Schnädelbach, Herbert: «Max Horkheimer und die Moralphilosophie des deutschen Idealismus», en Alfred Schmidt y Norbert Altwickler (eds.), Max Horkheiner heute: Werk und Wirkung, Francfort, 1986, pp. 52 y ss.

13. Cfr. Dewey, John: "The Theory of Emotion", Pars I, en Psychological Review, 1984, pp. 553 y ss.; Pars Ш, en Psychological Review, 1985, pp. 13 y ss.; sobre la teoría de los sentimientos de Dewey cfr. la muy enriquecedora aportación de Eduard Baumgarten, aDie geistigen Grundlagen des Amerikanischen Gemeinwesens», en Der Pragmatismus: RW. Emerson, W. James, J. Dewey, vol. II, Francfort, 1938, pp. 247 y ss.

14. Cfr. Piers, Gerhart/Singer, Milton B.: Shame and Guilt. A Psychoanalytic and a Cultural Study, Nueva York, 1971, especialmente pp. 23 y ss; Lynd, Helen M.: On Shame and the Search for Identity, Nucva York, 1958, cap. 2; tambiên Georg Simmel tiene una pequeña aportación sobre la cuestión presente: "Zur Psychologie der Scham" (1901), en Schriften zur Soziologie, editado por H.-J. Dahme y O. Rammstedt, Francfort, 1983, pp. 140 y ss. 